\title{
An Energy-Harvesting Device Based on Mechano-Electrospun Aligned Poly(vinylidene fluoride) Fiber Arrays
}

\author{
Ningbin Bu, YongAn Huang, ${ }^{*}$ Yajiang Ding, and Zhouping Yin ${ }^{* *}$ \\ State Key Laboratory of Digital Manufacturing Equipment and Technology, \\ Huazhong University of Science and Technology, Wuhan 430074, China
}

(Received July 23, 2015; accepted December 24, 2015)

Keywords: electrospinning, harvesting sensor, PVDF fiber

A nanogenerator is fabricated on the basis of in situ mechanical stretching and electrical poling of polyvinylidene fluoride fiber arrays deposited by mechano-electrospinning. The fibers with uniform polarization directions via loop motion control are directly written on flexible substrates with interdigitated comb electrodes. After encapsulation, the output voltage and current are enhanced compared with those in the postpoling treatment of near-field electrospinning fibers. The fabrication process reported here can be used to fabricate various energy-harvesting devices or actuators in the future.

\section{Introduction}

Energy-harvesting devices have been intensively studied owing to the global energy crisis. Recently, harvesting energy from tiny physical motion by nanogenerator-based nanofibers has been demonstrated as an excellent route for powering implantable devices and wearable electronics. ${ }^{(1-4)}$ Polyvinylidene fluoride (PVDF), which is a stable, low-cost, and environment-friendly polymer with high piezoelectricity, has been utilized in energy harvesters. ${ }^{(5)}$ By appropriate mechanical stretching and electrical poling, PVDF with good piezoelectric properties is achieved. ${ }^{(6,7)}$ The popular electrospinning technology can construct PVDF fibers simultaneously in one step by combining mechanical stretching and electrical poling.(8,9) However, fiber mats fabricated by conventional electrospinning do not exhibit useful piezoelectric responses as a whole. Since the piezoelectric reactions of randomly distributed fibers counteract each other, overall piezoelectric outputs are negligible.

The preparation of PVDF nanofibers by electrospinning with highly oriented and aligned structures has attracted considerable interest owing to enhanced electric outputs. Among the available methods, direct-write electrospinning technology is desirable for high controllability for satisfying the requirements of highly oriented structures. ${ }^{(5,10)}$ Nanogenerators have been produced using a single straight PVDF fiber by near-field electrospinning. ${ }^{(7,11)}$ After that, electrospun PVDF fiber webs have been arranged in a series of interdigitated comb electrodes to enhance the efficiency of energy harvesting. ${ }^{(12)}$ However, the postpoling treatment is necessary to prevent the partial reverse generation of neighboring electrodes. Moreover, the low voltage and stage moving speed are adopted in the near-field electrospining, which makes electric poling and stretching insufficient.

"Corresponding author: e-mail: yahuang@hust.edu.cn

${ }^{* *}$ Corresponding author: e-mail:yinzhp@mail.hust.edu.cn 
To obtain nanogenerators of higher performance, another electrospinning direct-write method called mechano-electrospinning (MES) may be an alternative method. This method utilizes the phenomenon whereby a charged jetted liquid fiber is pulled by a constant electric field force and a tunable mechanical drawing force at a longer nozzle-to-collector distance. Higher applied voltage and substrate moving speed can be achieved in the fabrication of different patterns. Hence, more $\beta$ phase of PVDF would be produced by this method. In this study, a nanogenerator is fabricated on the basis of the directly written fiber arrays by adopting MES in a continuously tunable manner. The effect of substrate moving velocity on the crystallinity of PVDF is presented. Finally, the nanogenerator is used to harvest mechanical energy from bending motion.

\section{Design and Fabrication of Device}

\subsection{Design of energy-harvesting device}

Figure 1(a) illustrates the structure of the fabricated nanogenerator. The PVDF nanofiber arrays are deposited on interdigitated comb electrodes on a polyethylene terephthalate (PET) sheet of 100 $\mu \mathrm{m}$ thickness. To facilitate the electric test of the harvesting device, a large pad is connected to the measurement device [a semiconductor characterization system (Keithley 4200-SCS) and a probe system (Cascade Summit 11000)] at the end of the electrode. On the device, an elastomer frame [polydimethylsiloxane (PDMS)] is used to cover the outer surface of a PVDF nanofiber array to support the recovery of the deformed device. Figure 1(b) shows the generation mechanism of the PVDF nanogenerator. In the free state, straight PVDF nanofibers are in the horizontal direction. When the PVDF nanofibers are bent, charges are generated by the combined tensile and bending stresses in the PVDF nanofibers, which results in electrical potentials between two adjacent electrodes. ${ }^{(1)}$ To prevent the second voltage poling and enhance the power output, the PVDF nanofibers on interdigitated electrodes are cut off one by one. This means that each pair of adjacent electrodes serve as unit cells and are connected in parallel.

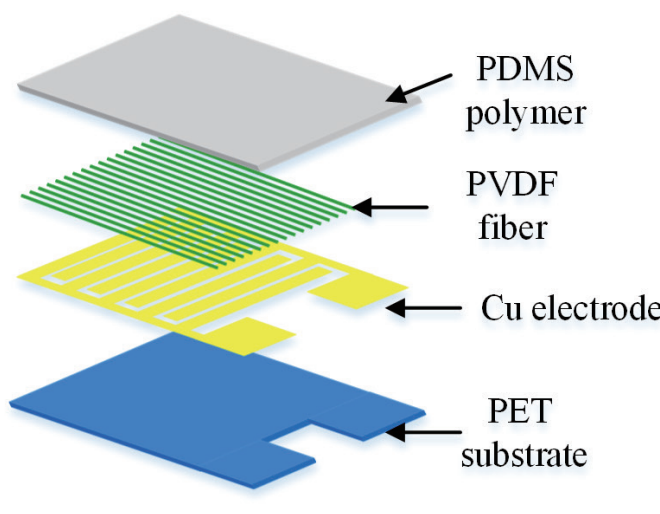

(a)
Free state

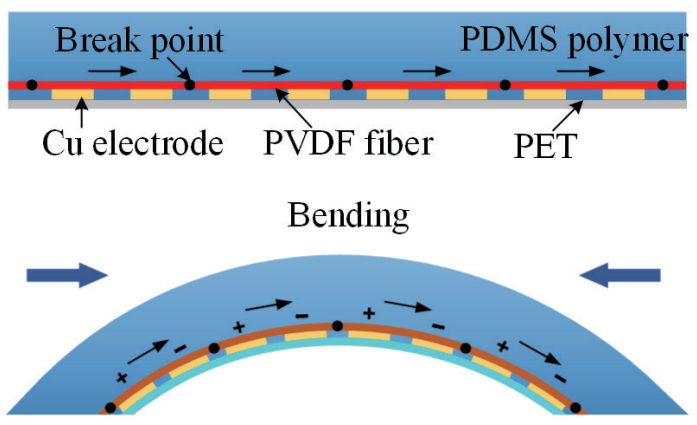

(b)

Fig. 1. (Color online) (a) Schematic of the construction of the PVDF nanogenerator. (b) Power generation mechanism of the PVDF nanogenerator. 


\subsection{Fabrication of energy-harvesting device}

Figure 2 show the fabrication process for the nanogenerator. First, copper adhesive tapes $(\mathrm{Cu}$ film thickness of $\sim 20 \mu \mathrm{m}$ ) cut into small rectangles are attached on top of a flexible PET film substrate of $100 \mu \mathrm{m}$ thickness [Fig. 2(a)]. Then, the resist is coated on the surface of the $\mathrm{Cu}$ film [Fig. 2(b)]. By UV lithography and development, interdigitated comb electrodes are formed [Figs. 2(c)-2(d)]. After that, the substrate is fixed on the moving stage and nanofibers are deposited on top of the film substrate by the designed moving trajectory [Fig. 2(e)]. To guarantee the uniform crystallinity direction of the PVDF, as the arrows indicated, the fiber arrays are formed by a continuous loop in the deposition process, which is presented in the inset figure. Finally, the fibers are cut and encapsulated with a PDMS film of $1 \mathrm{~mm}$ thickness [Fig. 2(f)].

The fiber arrays are deposited by mechano-electrospinning as shown in Fig. 2(e). The setup is used as previously reported. ${ }^{(13,14)}$ To fabricate periodic structures in a continuous and controllable manner, a programmable moving stage is used to carry the substrate. A stainless nozzle (inner diameter $(R)$ of $80 \mu \mathrm{m})$ is mounted vertically on the substrate. The solution is prepared by dissolving polyvinylidene fluoride (PVDF, Kynar 761) into dimethylformamide (DMF) and acetone (solvent ratio DMF:acetone, 6:4; Sigma-Aldrich). Then, the mixture $W_{\mathrm{t}}=18 \%$ is dispersed for 6 $\mathrm{h}$ using a magnetic stirrer. After standing for $1 \mathrm{~h}$, the solution is ready for electrospinning. In the experiments, the volume flow rate is $50 \mathrm{nl} / \mathrm{min}$ and the applied voltage decreases to $1.25 \mathrm{kV}$ after initiating the jet. The nozzle-to-substrate distance is fixed at $5 \mathrm{~mm}$.

After fabricating the electrodes and PVDF fibers, the device is encapsulated with a thin PDMS film, as shown in Fig. 2(f). The PDMS substrate (Sylgard 184, Dow Corning, Inc.) of $1 \mathrm{~mm}$ thickness was prepared by mixing the base and the curing agent at the ratio of 10:1. The mixture was first placed in a vacuum oven to remove air bubbles and then thermally cured at $70{ }^{\circ} \mathrm{C}$ for a short time, which makes the PDMS in a semicuring state. The substrate used was fresh and sticky.

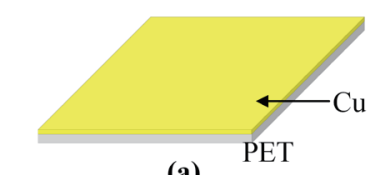

(a)

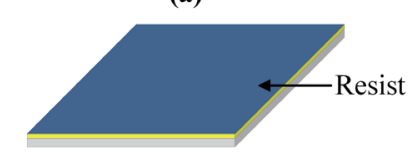

(b)

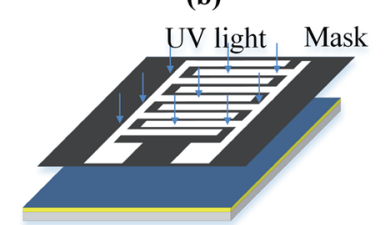

(c)

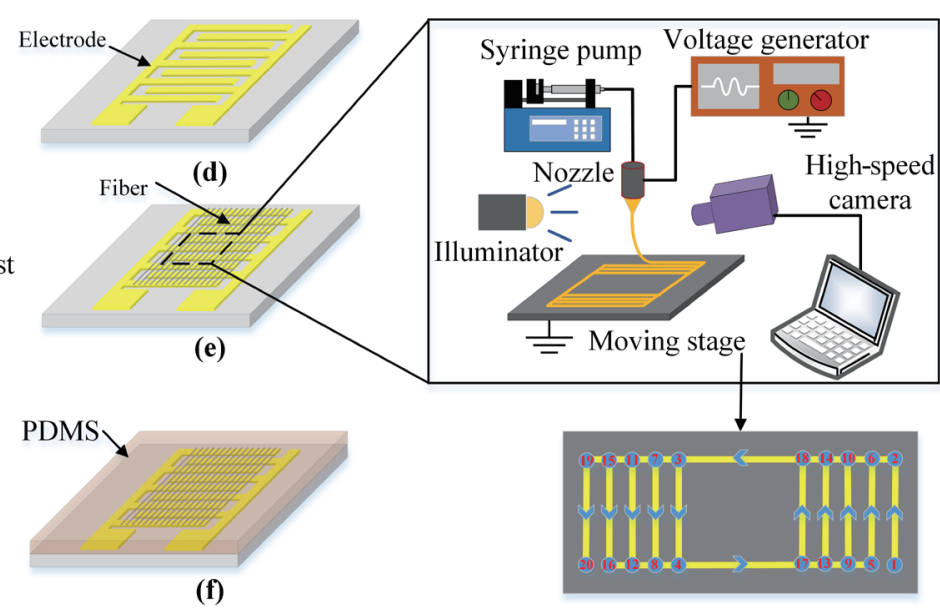

(f)

Fig. 2. (Color online) Schematic diagram of device fabrication process. (a) $\mathrm{Cu}$ tab adhesive. (b) Resist layer coating. (c) UV exposure and development. (d) Etching and resist removal. (e) Fiber deposition and breakage. (f) Encapsulation. 
Next, the PDMS layer was put directly on the PVDF fibers, which were deposited on the PET and thermally cured at $70{ }^{\circ} \mathrm{C}$ for $50 \mathrm{~min}$. Finally, we cut out the redundant PDMS around the harvesting device.

\section{Results and Discussion}

PVDF is a semicrystalline polymer with four crystalline phases, but only the $\beta$ phase exhibits dipole moments with piezoelectricity because the $\gamma$ and $\delta$ phases in the deposited fibers are so small that we can ignore their piezoelectricities in this situation. The pulling force originating from the moving substrate is important in the polarization of PVDF for obtaining more $\beta$ phase as a uniaxial tension process. To investigate the effect of the moving velocity of the substrate on the polarization, the fiber arrays with uniform orientation are also deposited on a silicon substrate at substrate velocities of 100,200 , and $300 \mathrm{~mm} / \mathrm{s}$ when fixing the voltage at $1.7 \mathrm{kV}$. Figure 3 (a) shows a fiber of $\sim 3 \mu \mathrm{m}$ diameter at a substrate velocity of $300 \mathrm{~mm} / \mathrm{s}$. From this figure, one can see that the fiber is attached on the surface more firmly due to the incomplete solvent evaporation at a short nozzle-to-collector distance. To characterize the crystal phases present in the PVDF nanofibers, Fig. 3(b) shows the Fourier transform infrared spectroscopy (FTIR) transmission spectrum of PVDF. One can indicate that polar $\beta$ phase bands appear distinctly at $511,600,840$, and $1275 \mathrm{~cm}^{-1}$. (15,16) Accordingly, the nonpolar $\alpha$ bands appear faintly at 411,795 , and $1402 \mathrm{~cm}^{-1}$. To determine the fraction of the content of the $\beta$ phase at different velocities, infrared spectroscopy absorption bands at 795 and $840 \mathrm{~cm}^{-1}$ are characterized as the $\alpha$ and $\beta$ phases, respectively. The relative fraction of the $\beta$ phase, $F(\beta)$, can be calculated using the following equation: ${ }^{(17)}$
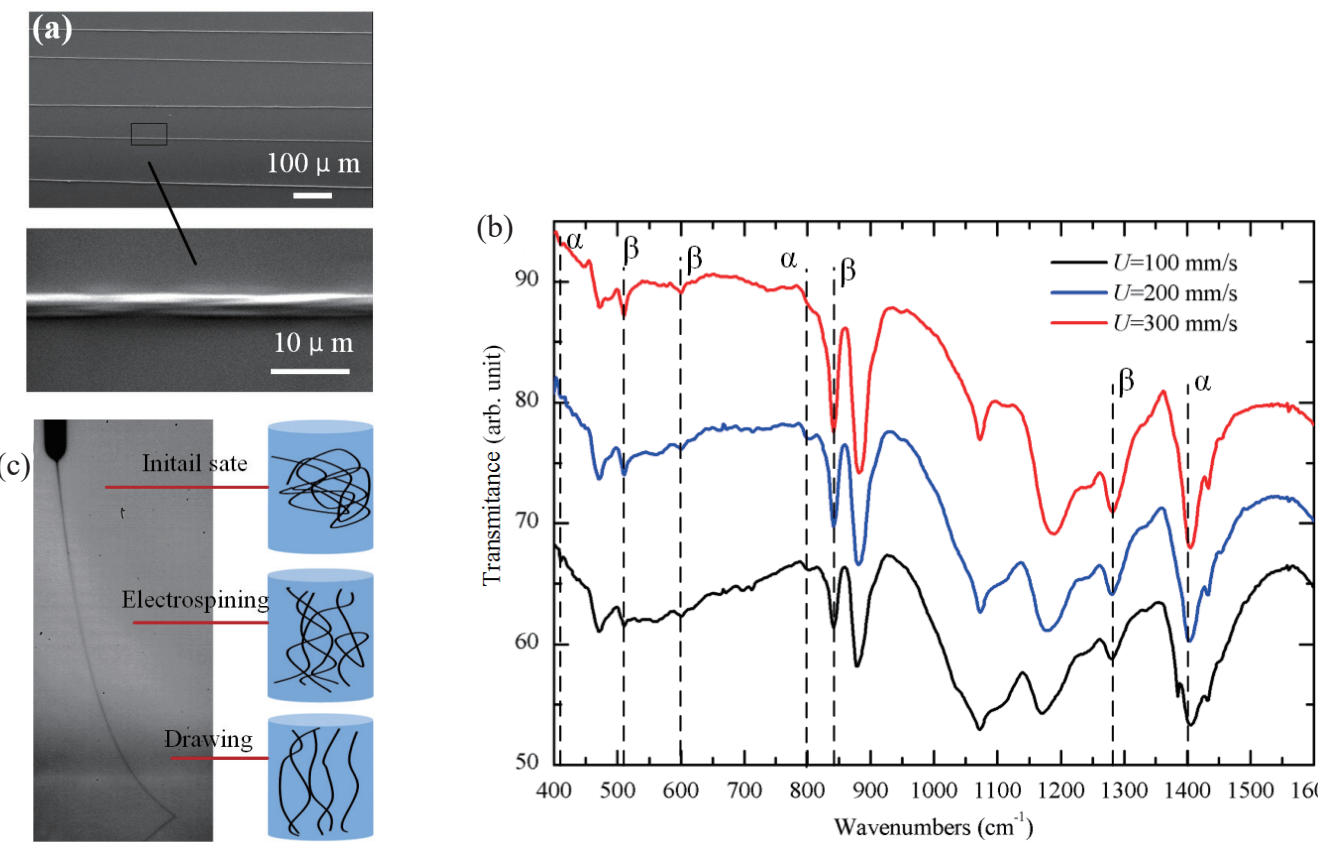

Fig. 3. (Color online) (a) Parallel nanofibers deposited by mechano-electrospinning. (b) FTIR transmission spectrum of PVDF nanofibers. (c) Schematic representation of the evolution of the fiber alignment and crystalline orientation based on jet deposition. 


$$
F(\beta)=\frac{X_{\beta}}{X_{\alpha}+X_{\beta}}=\frac{A_{\beta}}{\left(K_{\beta} / K_{\alpha}\right) A_{\alpha}+A_{\beta}}=\frac{A_{\beta}}{1.26 A_{\alpha}+A_{\beta}} .
$$

Here, $K_{\alpha}$ is $6.1 \times 10^{4}$ and $K_{\beta}$ is $7.7 \times 10^{4} \mathrm{~cm}^{2} / \mathrm{mol}$. The $A_{\alpha}$ and $A_{\beta}$ used in the calculation equation of the $\beta$ phase fraction correspond to absorption bands at 795 and $840 \mathrm{~cm}^{-1}$ for $\alpha$ and $\beta$ phases, respectively. Correspondingly, the variations of $F(\beta)$ are $48.45,52.4$, and $62.39 \%$ at 100,200 , and $300 \mathrm{~mm} / \mathrm{s}$, respectively. These results indicate that a high velocity enhances the transmission of the phase to the $\beta$ phase. The mechanism during the processing of the electrospun fiber is illustrated in Fig. 3(c). In the Taylor cone, the PVDF chain mass without any order is initially formed. For the drawing effect, note that as the jet approaches the substrate, the moving substrate adheres to the jet tail and accelerates the jet flow. By the interaction between the flow and the chain, the mass chain is drawn and aligned to the long planar conformation straight structure (the $\beta$ phase). For the poling effect, the cells of the $\alpha$ phase chain rotate to align their dipole moments in the direction favored by the external electric field, which increases the $\beta$ phase content. As the velocity increases, the drawing force increases as proved by the more curved jet trajectory. ${ }^{(13)}$

Figure 4(a) shows optical images of a fabricated device after the encapsulation of PDMS and the fiber on the gap between the two adjunct electrodes. The scanning electron microscopy (SEM) image of the single PVDF electrospun nanofiber indicates a diameter of $1 \mu \mathrm{m}$ at a voltage of $1.6 \mathrm{kV}$ and a velocity of $400 \mathrm{~mm} / \mathrm{s}$. In this device, the span of all the electrodes is $25 \mathrm{~mm}$ and the gap is $200 \mu \mathrm{m}$. Figure 4(b) shows the responses of the device under $1 \mathrm{~Hz}$ from a crank-slider mechanism with a work stroke of $12 \mathrm{~mm}$. By controlling the bend-release cycle for a given work stroke, the cycle frequency is directly related to the strain rate. The cycle frequencies range from 0.8 to 3.3
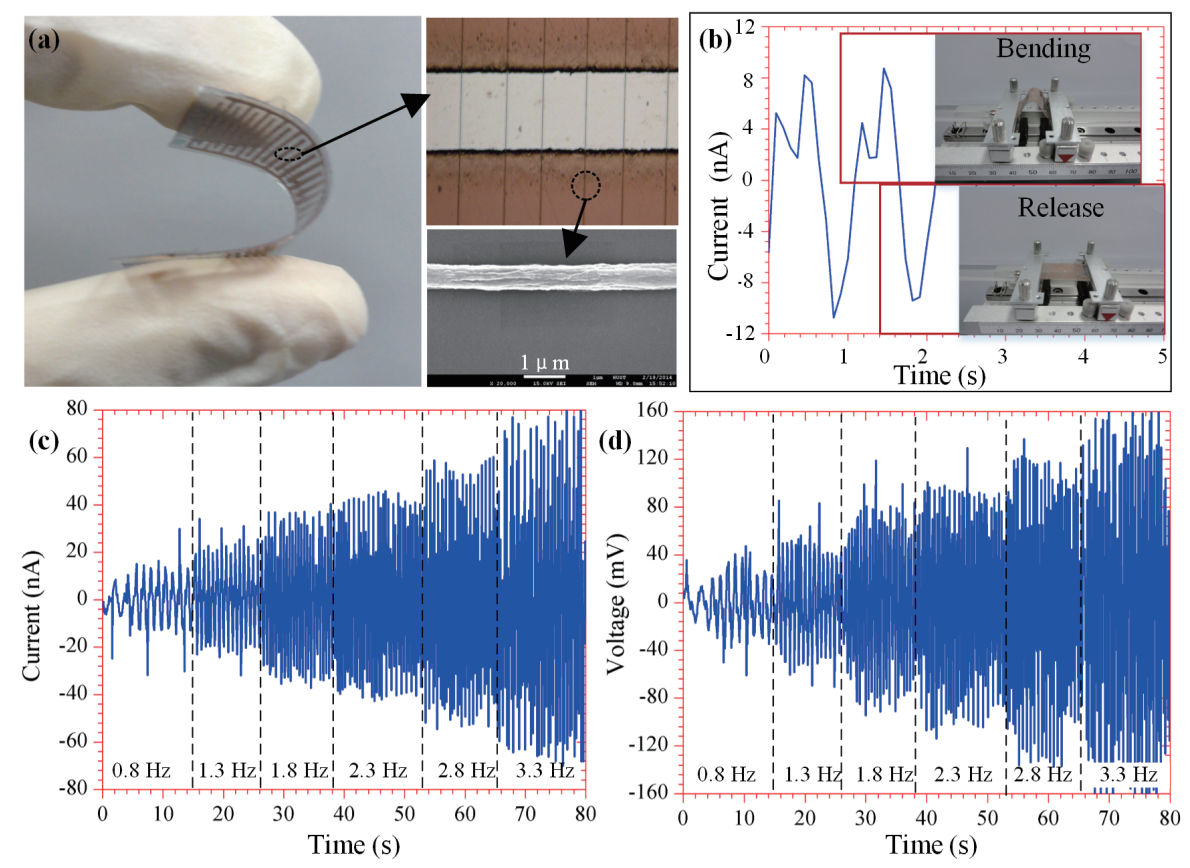

Fig. 4. (Color online) (a) Optical images of a fabricated nanogenerator and the fiber on the gap. (b) Measured current response of a PVDF fiber array under a cycle bending frequency of $1 \mathrm{~Hz}$. (c) Output current and (d) voltage under different cycle bending frequencies of $0.8-3.3 \mathrm{~Hz}$. 
$\mathrm{Hz}$ for the same work stroke of $12 \mathrm{~mm}$ [Figs. 4(c)-4(d)]. Measurement results show that the output currents and voltages increase proportionally as the cycle frequency increases during the bendrelease cycles. The average output currents of the peak are $\sim 10,20,32,40,52$, and $68 \mathrm{nA}$ in the cycle frequency ranges from 0.8 to $3.3 \mathrm{~Hz}$ and the output voltages are 24, 40,56, 88, 104, and $128 \mathrm{mV}$, respectively, which are consistent with the fundamental piezoelectric theory, that is, a high strain rate leads to a linear scale up of output voltages and currents. ${ }^{(18)}$ At the same time, the maximum cycle frequency for the device is $4 \mathrm{~Hz}$. We can only get the $50 \mathrm{nA}$ current in the case of $4 \mathrm{~Hz}$, and the output currents fluctuate heavily because the PDMS cannot be released fully and uniformly at such a high frequency.

\section{Summary and Conclusions}

In summary, we demonstrate a simple nanogenerator configuration with a simple fabrication process for harvesting energy in a tiny motion. By a mechano-electrospining direct-write method, fiber arrays with $\beta$-phase are fabricated in one polarization direction on a flexible substrate. Moreover, in situ mechanical stretching and electrical poling are able to induce more $\beta$ phase from the original $\alpha$ phase. A higher moving velocity could lead to the generation of more $\beta$-phase owing to a stronger drawing force. The output voltages and currents are scaled up by the comb electrode in a bend-release cycle. In the future, this fabrication method can be used to fabricate various sensors, energy-harvesting devices or actuators based on different piezoelectric fibers.

\section{Acknowledgements}

The authors acknowledge support from the National Natural Science Foundation of China (51035002 and 51175209) and the Fundamental Research Funds for the Central Universities (2011TS024). The general characterization facilities were provided by the National Laboratory.

\section{References}

1 X. Chen, S. Xu, N. Yao, and Y. Shi: Nano Lett. 10 (2010) 2133.

2 J. Fang, X. Wang, and T. Lin: J. Mater. Chem. 21 (2011) 11088.

3 B. J. Hansen, Y. Liu, R. Yang, and Z. L. Wang: ACS Nano 4 (2010) 3647.

4 H. Henrichsen, H. Sturm, P. Bøggild, and O. Hansen: J. Vac. Sci. Technol. B 28 (2010) 617.

5 Y. Huang, N. Bu, Y. Duan, Y. Pan, H. Liu, Z. Yin, and Y. Xiong: Nanoscale 5 (2013) 12007.

6 S. Najarian, J. Dargahi, and V. Mirjalili: Sens. Mater. 18 (2006) 215.

7 C. T. Pan, C. K. Yen, Z. H. Liu, H. W. Li, S. W. Kuo, Y. S. Lu, and Y. C. Lai: Sens. Mater. 26 (2014) 63.

8 H. Yu, T. Huang, M. Lu, M. Mao, Q. Zhang, and H. Wang: Nanotechnology 24 (2013) 405401.

9 J. Fang, H. Niu, H. Wang, X. Wang, and T. Lin: Energy Environ. Sci. 6 (2013) 2196.

10 M. Rinaldi, F. Ruggieri, L. Lozzi, and S. Santucci: J. Vac. Sci. Technol. B 27 (2009) 1829.

11 J. Pu, X. Yan, Y. Jiang, C. Chang, and L. Lin: Sens. Actuators, A 164 (2010) 131.

12 J. Chang and L. Lin: Solid-State Sens., IEEE Actuators Microsyst. Conf. (TRANSDUCERS) (2011) p. 747.

13 Y. Huang, Y. Duan, Y. Ding, N. Bu, Y. Pan, N. Lu, and Z. Yin: Sci. Rep. 4 (2014) 5949.

14 Y. Duan, Y. Huang, Z. Yin, N. Bu, and W. Dong: Nanoscale 5 (2013) 12007.

15 M. V. Moreira, S. Lanceros-Méndez, R. Gregório Filho, A. S. Pouzada, and V. Sencadas: Mater. Sci. Forum (2006) 872.

16 J. Andrew and D. Clarke: Langmuir 24 (2008) 670.

17 M. Ramos, H. M. Correia, and S. Lanceros-Mendez: Comput. Mater. Sci. 33 (2005) 230.

18 Y. K. Fuh, S. Y. Chen, and J. C. Ye: Appl. Phys. Lett. 103 (2013) 033114. 